\title{
A Reliable Split-Step Fourier Method for the Propagation Equation of Ultra-Fast Pulses in Single-Mode Optical Fibers
}

\author{
Ralf Deiterding, Roland Glowinski, Hilde Oliver, and Stephen Poole Member, IEEE
}

\begin{abstract}
The extension to the split-step Fourier method (SSFM) for Schrödinger-type pulse propagation equations that we propose in this article is designed with the accurate simulation of pulses in the femto-second regime in single-mode communication fibers in mind. We show that via an appropriate operator splitting scheme, Kerr nonlinearity and the self-steepening and stimulated Raman scattering terms can be combined into a single sub-step consisting of an inhomogeneous quasilinear first-order hyperbolic system for the real-valued quantities intensity and phase. First- and second-order accurate shock-capturing upwind schemes have been developed specifically for this nonlinear substep, which enables the accurate and oscillation-free simulation of signals under the influence of Raman scattering and extreme selfsteepening with the SSFM. Benchmark computations of ultra-fast Gaussian pulses in fibers with strong nonlinearity demonstrate the superior approximation properties of the proposed approach.
\end{abstract}

Index Terms-Fiber optical communication, ultra-fast Gaussian pulse, self-steepening, Raman scattering, split-step Fourier method (SSFM), shock-capturing upwind scheme

\section{INTRODUCTION}

$\mathbf{T}$ HE propagation of ultra-short pulses in single-mode communication fibers is typically modeled with the generalized nonlinear Schrödinger equation [1], [2] by

$$
\begin{aligned}
\frac{\partial A}{\partial z}+ & \frac{\alpha}{2} A+i \frac{\beta_{2}}{2} \frac{\partial^{2} A}{\partial T^{2}}-\frac{\beta_{3}}{6} \frac{\partial^{3} A}{\partial T^{3}} \\
& =i \gamma\left(A|A|^{2}+i S \frac{\partial}{\partial T}\left(A|A|^{2}\right)-T_{R} A \frac{\partial|A|^{2}}{\partial T}\right)
\end{aligned}
$$

In (1), $A(z, T)$ denotes the slowly varying field envelope of the signal and $z$ is the propagation distance. Equation (1) is valid in the frame of reference of the pulse traveling with signal group velocity $v_{g}$. Using the relation $T=t-z / v_{g}$ between the retarded time $T$ and the present time $t$, (1) can be transformed into the laboratory frame of reference. The parameter $\alpha$ models linear signal loss, $\beta_{2}$ and $\beta_{3}$ are the coefficients of second- and third-order dispersion and $\gamma$ is the nonlinear fiber parameter. The parameters $S$ and $T_{R}$ govern, respectively, the effects of self-steepening and stimulated Raman scattering.

R. Deiterding and S. Poole are with the Computer Science and Mathematics Division of Oak Ridge National Laboratory, P.O. Box 2008 MS6164, Oak Ridge, TN 37831 USA e-mail: deiterdingr@ornl.gov.

R. Glowinski is with the Department of Mathematics, University of Houston, 651 P. G. Hoffman Hall, Houston, TX 77204-3008 USA.

H. Oliver is with the Department of Mathematics, University of South Carolina, 1523 Greene Street, Columbia, SC 29208 USA.

Manuscript received *
Since analytic solutions of (1) are generally not available, numerical solution techniques are of fundamental importance for modeling pulse propagation. The dominant approach consists of split-step schemes that perform spatial sub-steps considering only the linearities on the left-hand side of (1) by discrete Fourier transformation and sub-steps approximating only the influence of the nonlinear right-hand side terms in an alternating fashion. When pulse widths are well in the pico-second regime, $S=0$ and $T_{R}=0$ can be used and all nonlinear derivatives vanish. For this specific regime, the construction of such split-step Fourier methods (SSFM) is very well established, cf. [1], [3]. However, the topic of how to incorporate both self-steepening and Raman scattering reliably into the SSFM, as it is required for the predictive simulation of femto-second pulses, which will occur for instance in $\mathrm{Tb} / \mathrm{s}$ communication networks, has received little attention.

One presently popular approach approximates (1) similarly to the general pulse propagation equation

$$
\begin{array}{r}
\frac{\partial A}{\partial z}+\frac{\alpha}{2} A+\left(\sum_{k \geq 2} \beta_{k} \frac{i^{k-1}}{k !} \frac{\partial^{k}}{\partial T^{k}}\right) A=i \gamma\left(1+i S \frac{\partial}{\partial T}\right) \\
\times\left[A \int_{-\infty}^{\infty} R\left(T^{\prime}\right)\left|A\left(T-T^{\prime}\right)\right|^{2} d T^{\prime}\right]
\end{array}
$$

from which it has been derived assuming self-steepening and stimulated Raman scattering can be modeled independently (see [1] for details). Because of the convolution integral, the nonlinear right-hand side of (2) seems amenable only to element-wise forward and inverse Fourier transformations [4]. So far, photonics researchers seem to have been content applying the same technique to the right-hand side of (1), cf. [2], [5]. Here, however, we will describe a new and alternative class of SSFM, which is based on a nonlinear operator constructed specifically for (1), that is characterized by unprecedented robustness. In particular, our proposed new approach handles both extreme self-steepening and stimulated Raman scattering reliably.

This paper is organized as follows: In Section II, we will recall the construction principles of split-step Fourier methods detailing available spatial update algorithms and the linear sub-step. In Section III, special attention will be devoted to the development of possible nonlinear sub-operators tailored for (1). We will show how the complex-valued right-hand side of (1) can be transformed into a first-order system of quasilinear partial differential equations for the real-valued 
quantities intensity and phase. It is easily verified that this sub-system is of hyperbolic type. Based on this observation, we will construct specially tailored high-resolution shockcapturing discretizations for this system borrowing from techniques originally developed in the context of hydrodynamics. Note that our derivations take advantage of the structure of (1) and are not directly applicable to the more general model (2). Benchmarks in Section IV will demonstrate that the resulting overall SSFM for (1) is of second-order numerical accuracy, with an absolute error below any other tested second-order scheme, and able to simulate pulse propagation close (and even beyond) the point of optical shock formation with consistent convergence. The conclusions will follow in Section V.

\section{SPLIT-STEP FOURIER SCHEMES}

\section{A. Splitting algorithms}

In order to develop a numerical solution method for (1) we write the equation in the form

$$
\begin{aligned}
\frac{\partial A}{\partial z}= & \underbrace{\left(-\frac{\alpha}{2}-i \frac{\beta_{2}}{2} \frac{\partial^{2}}{\partial T^{2}}+\frac{\beta_{3}}{6} \frac{\partial^{3}}{\partial T^{3}}\right)}_{\mathcal{D}} A \\
& +\underbrace{i \gamma\left(|A|^{2}+i S \frac{1}{A} \frac{\partial}{\partial T}\left(A|A|^{2}\right)-T_{R} \frac{\partial|A|^{2}}{\partial T}\right)}_{\mathcal{N}} A,
\end{aligned}
$$

where we denote with $\mathcal{D}(A)$ the operator of all terms linear in $A$ and with $\mathcal{N}(A)$ the operator of all nonlinearities. Using these definitions, we write (3) in short as

$$
\frac{\partial A}{\partial z}=(\mathcal{D}+\mathcal{N}) A .
$$

If one assumes $\mathcal{D}$ and $\mathcal{N}$ to be independent of $z$, (4) can be integrated exactly and the solution at $z+h$ reads

$$
A(z+h, T)=\exp (h(\mathcal{D}+\mathcal{N})) A(z, T) .
$$

The last expression forms the basis of split-step numerical methods [1]. Note, however, that the operators $\mathcal{D}$ and $\mathcal{N}$ in general do not commute and that it corresponds to an $O(h)$ approximation to replace (5) with $\exp (h \mathcal{D}) \exp (h \mathcal{N}) A(z, T)$. A commonly used symmetric approximation is [6], [7]

$$
A(z+h, T)=\exp \left(\frac{h}{2} \mathcal{D}\right) \exp (h \mathcal{N}) \exp \left(\frac{h}{2} \mathcal{D}\right) A(z, T) .
$$

Utilizing the Baker-Campbell-Hausdorff formula for expanding two non-commuting operators, (6) can be proven to be an $O\left(h^{2}\right)$ approximation [8]. Comprehensive descriptions of the split-step approach for simulating pulse propagation in fibers are given for instance by Agrawal [1] and Hohage $\&$ Schmidt [3]. The efficiency of the SSFM, especially for longer propagation distances as required for modeling optical communication lines, can be improved by taking solution adaptive steps in space as proposed by Sinkin et al. [9].

While (6) leads to numerical methods that are maximally second-order accurate [3], the utilization of explicit RungeKutta methods for the spatial update is also possible [4], [10].
Especially notably is the interaction picture (IAP) algorithm by Hult [11] of $O\left(h^{4}\right)$ accuracy that reads

$$
\begin{aligned}
A_{I} & =\exp \left(\frac{h}{2} \mathcal{D}\right) A(z, T), \\
k_{1} & =\exp \left(\frac{h}{2} \mathcal{D}\right)[h \mathcal{N}(A(z, T))] A(z, T), \\
k_{2} & =h \mathcal{N}\left(A_{I}+k_{1} / 2\right)\left[A_{I}+k_{1} / 2\right], \\
k_{3} & =h \mathcal{N}\left(A_{I}+k_{2} / 2\right)\left[A_{I}+k_{2} / 2\right], \\
k_{4} & =h \mathcal{N}\left(\exp \left(\frac{h}{2} \mathcal{D}\right)\left(A_{I}+k_{3}\right)\right)\left[\exp \left(\frac{h}{2} \mathcal{D}\right)\left(A_{I}+k_{3}\right)\right], \\
A(z+h, T) & =\exp \left(\frac{h}{2} \mathcal{D}\right)\left[A_{I}+\frac{k_{1}}{6}+\frac{k_{2}}{3}+\frac{k_{3}}{3}\right]+\frac{k_{4}}{6} .
\end{aligned}
$$

The benefit of the IAP method versus a straightforward fourstep Runge-Kutta implementation [4], [10] is the reduction of required applications of the linear sub-operator $\mathcal{D}$ from 8 down to 4 . The combination of the IAP algorithm with spatial adaptation has been suggested by Liu [12].

\section{B. Linear sub-steps}

Since the dispersion coefficients $\beta_{2}$ and $\beta_{3}$ are generally small compared to the other problem parameters, leading to numerical stiffness, discretization of the temporal derivatives in $\mathcal{D}$ by finite differences would result in a numerical method with very high resolution requirements. Instead, it is computationally more efficient to apply Fourier transformation in $T$ to $\mathcal{D}$ and transform the differentials into multiplications in frequency space. For (1) the linear operator becomes

$$
\begin{aligned}
& \exp \left(\frac{h}{2} \mathcal{D}\right) A(z, T) \\
& =\mathcal{F}^{-1} \exp \left[\frac{h}{2}\left(i \frac{\beta_{2}}{2} \omega^{2}-i \frac{\beta_{3}}{6} \omega^{3}-\frac{\alpha}{2}\right)\right] \mathcal{F} A(z, T),
\end{aligned}
$$

where $\mathcal{F}$ and $\mathcal{F}^{-1}$ denote Fourier and inverse Fourier transformation, respectively. Note that it is straightforward to consider higher-order linear derivatives as indicated in the left-hand side of (2) in the same manner.

In a practical computer program, one uses discrete Fourier transformation and for $\omega$ we employ the discrete frequency spectrum $\{j \omega: j \in \mathbb{Z},-N \leq j \leq N-1\}$ with spectral width $\Delta \omega=\pi /(N \Delta T)$. In doing so, it is assumed that the temporal window traveling with the pulse is discretized with $2 N$ points. ${ }^{1}$ Denoting the temporal discretization width with $\Delta T$, this gives a temporal window of the extensions $[N \Delta T,(N-1) \Delta T]$. $A_{j}$ denotes the numerical approximation to $A(\cdot, j \Delta T)$ at the discrete point with index $j$.

\section{NONLINEAR SUB-STEPS}

\section{A. Central difference method}

A comparably straightforward approach for deriving a nonlinear operator $\mathcal{N}(A)$ is to discretize all nonlinear differentials in retarded physical time. Utilizing $|A|^{2}=A \bar{A}$ we eliminate $1 / A$ from $\mathcal{N}(A)$, which we write as

$$
\mathcal{N}(A)=i \gamma\left(|A|^{2}+i S \bar{A} \frac{\partial A}{\partial T}+\left[i S-T_{R}\right] \frac{\partial|A|^{2}}{\partial T}\right) .
$$

\footnotetext{
${ }^{1}$ Note that fast Fourier transformation algorithms are specially efficient if $N$ is a power of 2 .
} 
The simplest idea, is then to approximate the temporal derivatives in (9) with complex-valued second-order accurate central differences of the discrete values $A_{j}$, i.e.

$$
\begin{aligned}
\mathcal{N}(A) & =i \gamma\left(\left|A_{j}\right|^{2}+i S \bar{A}_{j} \frac{A_{j+1}-A_{j-1}}{2 \Delta T}\right. \\
& \left.+\left[i S-T_{R}\right] \frac{\left|A_{j+1}\right|^{2}-\left|A_{j-1}\right|^{2}}{2 \Delta T}\right)+O\left(\Delta T^{2}\right) .
\end{aligned}
$$

Consistent with the linear operator, (8), periodic boundary conditions are assumed. Here, we implement boundary conditions for the temporal window by employing auxiliary points outside of the actual domain (also called "halo" or "ghost" by some authors)

$$
A_{-N-\nu}:=A_{N-\nu}, \quad A_{N+\nu-1}:=A_{-N+\nu-1}
$$

for $\nu \in\{1, \ldots, p\}$, which are set immediately before the nonlinear update step. The implementation of operator (10) requires $p=1$.

Utilization of (10) to approximate $\exp (h \mathcal{N}(A))$ in the splitstep scheme (6) leads to a numerical method that is of overall second-order accuracy. When used within the fourth-oder accurate interaction picture algorithm (7), the $O\left(\Delta T^{2}\right)$ accuracy of (10) limits the overall accuracy to second order, although absolute errors are slightly reduced, cf. Section IV-A. It is also clear that the central differences in (10) can potentially result in numerical oscillations (aka Gibbs phenomena [13]), for instance when strong self-steepening occurs. Nevertheless, the apparent simplicity of the operator (10) makes it particularly suitable for verification and comparison with the schemes proposed below in the Sections III-C and III-D.

\section{B. Fourier transformation}

A presently popular approach for handling $\mathcal{N}(A)$ is to apply forward and inverse Fourier transformation individually to the derivatives, cf. [2]. For instance, in (9) one simply replaces $\bar{A} \partial_{T} A$ with $\bar{A} \mathcal{F}^{-1}(i \omega \mathcal{F}(A))$, thereby neglecting the dependence of $\bar{A}$ on $T$. The result is a numerical operator that would generally not be consistent in the strict mathematical sense with $\mathcal{N}(A)$. For instance, we could transform (9) into

$$
\begin{aligned}
\mathcal{N}(A) \approx i \gamma\left(|A|^{2}+\right. & i S \bar{A} \mathcal{F}^{-1}(i \omega \mathcal{F}(A)) \\
& \left.+\left[i S-T_{R}\right] \mathcal{F}^{-1}\left(i \omega \mathcal{F}\left(|A|^{2}\right)\right)\right) .
\end{aligned}
$$

However, (12) is only an approximation to (9) and if we replace $|A|^{2}$ with $A \bar{A}$ in the differentials of (9) and apply the product rule to obtain

$$
\mathcal{N}(A)=i \gamma\left(|A|^{2}+\left[i S-T_{R}\right] A \frac{\partial \bar{A}}{\partial T}+\left[2 i S-T_{R}\right] \bar{A} \frac{\partial A}{\partial T}\right),
$$

the resulting approximate nonlinear operator would read

$$
\begin{array}{r}
\mathcal{N}(A) \approx i \gamma\left(|A|^{2}+\left[i S-T_{R}\right] A \mathcal{F}^{-1}(i \omega \mathcal{F}(\bar{A}))+\right. \\
\left.\left[2 i S-T_{R}\right] \bar{A} \mathcal{F}^{-1}(i \omega \mathcal{F}(A))\right) .
\end{array}
$$

The approximations (12) and (14) are not identical and thereby correspond to slightly different discretizations of (9). Basically (14) is used in the freely available software LaserFOAM
[5], while Long et al. [2] apply this technique directly to the right-hand side of (1). Although in combination with the IAP method (7) the sketched Fourier-transformation-based nonlinear operators can exhibit numerical fourth order convergence, cf. Section IV-A, the lack of strict mathematical consistency and apparent ambiguity in operator construction are concerning. Particularly, it is not obvious that a numerical approximation under grid convergence would correspond to a solution of the original differential equation (1). Further on, as will be demonstrated in Section IV, the Fouriertransformation-based approach struggles generically with large gradients requiring very high resolution in $T$ and produces serious Gibbs oscillations usually much earlier than the central difference operator (10). In here, we show results with (12) and (14) primarily for comparison.

\section{Upwind approach}

The nonlinear operators presented so far have in common that they can be used equally in the symmetric SSFM (6) and the IAP algorithm (7). Obviously, they treat the Kerr nonlinearity identically and are direct extensions of numerical methods originally published for the case $S=0$ and $T_{R}=0$. However, in order to derive alternative numerical methods for (1) we have opted to deviate from this path and avoid factoring out $A$ from the nonlinear operator. With a symmetric split-step method of the basic structure

$$
\begin{aligned}
A^{*} & =\exp \left(\frac{h}{2} \mathcal{D}\right) A(z, T), \\
A^{* *} & =A^{*}+h \overline{\mathcal{N}}\left(A^{*}\right), \\
A(z+h, T) & =\exp \left(\frac{h}{2} \mathcal{D}\right) A^{* *}
\end{aligned}
$$

in mind, our objective is to derive a numerical method that is optimally suited for the nonlinear sub-problem

$$
\frac{\partial A}{\partial z}=\underbrace{i \gamma\left(A|A|^{2}+i S \frac{\partial}{\partial T}\left(A|A|^{2}\right)-T_{R} A \frac{\partial|A|^{2}}{\partial T}\right)}_{\mathcal{N}(A)} .
$$

Note that step (15b) is written here as a simple explicit Euler method to motivate the fundamental idea but schemes described below are in fact more complicate.

Using the Madelung transformation [14], [15]

$$
A(z, t)=\sqrt{I(z, t)} \exp (i \phi(z, t)),
$$

we can transform (16) into the equivalent system of hydrodynamical partial differential equations

$$
\begin{gathered}
\frac{\partial I}{\partial z}+3 \gamma S I \frac{\partial I}{\partial T}=0, \\
\frac{\partial \phi}{\partial z}+\gamma S I \frac{\partial \phi}{\partial T}+\gamma T_{R} \frac{\partial I}{\partial T}=\gamma I
\end{gathered}
$$

of the real-valued quantities intensity $I(z, T)=|A(z, T)|^{2}$ and phase $\phi(z, T)=\arg (A(z, T))$. If we write (18) in the form

$$
\frac{\partial}{\partial z}\left[\begin{array}{l}
I \\
\phi
\end{array}\right]+\left[\begin{array}{cc}
3 \gamma S I & 0 \\
\gamma T_{R} & \gamma S I
\end{array}\right] \frac{\partial}{\partial T}\left[\begin{array}{l}
I \\
\phi
\end{array}\right]=\left[\begin{array}{c}
0 \\
\gamma I
\end{array}\right],
$$

its structure as an inhomogeneous quasilinear system

$$
\partial_{z} \mathbf{q}+\mathbf{M}(\mathbf{q}) \partial_{T} \mathbf{q}=\mathbf{s}(\mathbf{q})
$$


with $\mathbf{q}=(I, \phi)^{T}$ becomes apparent. If we assume $\gamma \neq 0$ and $S \neq 0$, the matrix $\mathbf{M}(\mathbf{q})$ has for $I \neq 0$ the eigendecomposition $\mathbf{M}=\mathbf{R} \boldsymbol{\Lambda} \mathbf{R}^{-1}$ with $\boldsymbol{\Lambda}=\operatorname{diag}(\gamma S I, 3 \gamma S I)$ and

$$
\mathbf{R}=\left[\begin{array}{cc}
0 & \frac{2 S I}{T_{R}} \\
1 & 1
\end{array}\right], \quad \mathbf{R}^{-1}=\left[\begin{array}{cc}
-\frac{T_{R}}{2 S I} & 1 \\
\frac{T_{R}}{2 S I} & 0
\end{array}\right]
$$

The eigenvalues $\lambda_{1}=\gamma S I$ and $\lambda_{2}=3 \gamma S I$ are distinct and the eigenvectors (column vectors of $\mathbf{R}$ ) are linearly independent, which proves the strict hyperbolicity of (19) for $I \neq 0$. Following the theory of hyperbolic equations, cf. [16], the solution of (19) is given as the quasilinear superposition of two waves traveling with the characteristic speeds $\lambda_{1}$ and $\lambda_{2}$. It is very well established, cf. [17], that numerical methods for hyperbolic problems benefit significantly if the direction of wave propagation, that is the sign of the eigenvalues, is considered in order to construct one-sided finite differences.

Again, we adopt an operator splitting technique and, instead of discretizing (20) directly, alternate between solving the homogeneous partial differential equation

$$
\partial_{z} \mathbf{q}+\mathbf{M}(\mathbf{q}) \partial_{T} \mathbf{q}=0
$$

and the ordinary differential equation

$$
\partial_{z} \mathbf{q}=\mathbf{s}(\mathbf{q})
$$

successively, using the updated data from the preceding step as initial condition. We index the spatial steps with $n \in \mathbb{N}_{0}$ and the temporal discretization points with $j$. In what follows, we employ the convention that differences between two adjacent discrete values are always indicated by the $\Delta$ symbol; algebraic averages of two neighboring quantities always receive the ^ superscript.

Utilizing the definitions $\Delta \mathbf{q}_{j+1 / 2}^{n}:=\mathbf{q}_{j+1}^{n}-\mathbf{q}_{j}^{n}$ and $\hat{\mathbf{q}}_{j+1 / 2}^{n}:=\frac{1}{2}\left(\mathbf{q}_{j+1}^{n}+\mathbf{q}_{j}^{n}\right)$, one can approximate (22) with the upwind formula

$$
\begin{aligned}
\mathbf{q}_{j}^{*}=\mathbf{q}_{j}^{n}-\frac{h}{\Delta T}\left(\hat{\mathbf{M}}^{-}\left(\mathbf{q}_{j+1}^{n}, \mathbf{q}_{j}^{n}\right) \Delta \mathbf{q}_{j+1 / 2}^{n}\right. & \\
& \left.+\hat{\mathbf{M}}^{+}\left(\mathbf{q}_{j}^{n}, \mathbf{q}_{j-1}^{n}\right) \Delta \mathbf{q}_{j-1 / 2}^{n}\right)
\end{aligned}
$$

and (23) with the subsequent forward Euler update

$$
\mathbf{q}_{j}^{n+1}=\mathbf{q}_{j}^{n}+h \mathbf{s}\left(\mathbf{q}_{\mathbf{j}}^{*}\right) .
$$

In the general case, the matrices $\hat{\mathbf{M}}^{+}$and $\hat{\mathbf{M}}^{-}$indicate decompositions of $\hat{\mathbf{M}}\left(\mathbf{q}_{j+1}^{n}, \mathbf{q}_{j}^{n}\right):=\mathbf{M}\left(\hat{\mathbf{q}}_{j+1 / 2}^{n}\right)$ with only positive and negative eigenvalues, respectively, that are constructed by specifying $\hat{\boldsymbol{\Lambda}}^{+}:=\operatorname{diag}\left(\max \left\{\hat{\lambda}_{m}, 0\right\}\right), \hat{\mathbf{\Lambda}}^{-}:=$ $\operatorname{diag}\left(\min \left\{\hat{\lambda}_{m}, 0\right\}\right)$ as $\hat{\mathbf{M}}^{ \pm}:=\hat{\mathbf{R}} \hat{\mathbf{\Lambda}}^{ \pm} \hat{\mathbf{R}}^{-1}$. With the last definition, $\hat{\mathbf{M}}^{ \pm} \Delta \mathbf{q}$ can be effectively computed as (suppressing indices $n$ and $j$ in the notation)

$$
\hat{\mathbf{M}}^{-} \Delta \mathbf{q}=\sum_{\substack{\hat{\lambda}_{m}<0 \\ m=\{1,2\}}} \hat{\lambda}_{m} a_{m} \hat{\mathbf{r}}_{m}, \quad \hat{\mathbf{M}}^{+} \Delta \mathbf{q}=\sum_{\substack{\hat{\lambda}_{m} \geq 0 \\ m=\{1,2\}}} \hat{\lambda}_{m} a_{m} \hat{\mathbf{r}}_{m},
$$

where $\hat{\mathbf{r}}_{m}$ denotes the eigenvectors averaged between two neighboring points and $a_{m}$ are the corresponding entries of the vector $\mathbf{a}=\hat{\mathbf{R}}^{-1} \Delta \mathbf{q}$ that for $\hat{\mathbf{R}}^{-1}$ of (21) reads

$$
\mathbf{a}=\left(\Delta \phi-\frac{T_{R} \Delta I}{2 S \hat{I}}, \frac{T_{R} \Delta I}{2 S \hat{I}}\right)^{T} .
$$

In the case of (19), however, the eigenvalues have the same sign, which depends solely on the sign of $\gamma$ (since $S \geq 0$ and $I \geq 0$ ), and we can use $\hat{\mathbf{M}}^{+}=\hat{\mathbf{M}}, \hat{\mathbf{M}}^{-}=0$ for $\gamma>0$, and $\hat{\mathbf{M}}^{+}=0, \hat{\mathbf{M}}^{-}=\hat{\mathbf{M}}$ for $\gamma<0$. Inserting the latter into (24) and (25) an upwind scheme for (19) is readily derived as

$$
\begin{aligned}
I_{j}^{n+1} & =I_{j}^{n}-\frac{h}{\Delta T}\left[3 \gamma S \tilde{I}_{j}^{n} \Delta I_{j}^{n}\right], \\
\tilde{\phi}_{j}^{n+1} & =\phi_{j}^{n}-\frac{h}{\Delta T}\left[\gamma T_{R} \Delta I_{j}^{n}+\gamma S \tilde{I}_{j}^{n} \Delta \phi_{j}^{n}\right], \\
\phi_{j}^{n+1} & =\tilde{\phi}_{j}^{n+1}+h \gamma I_{j}^{n+1}
\end{aligned}
$$

with

$$
\begin{array}{ll}
\Delta I_{j}^{n}=I_{j}^{n}-I_{j-1}^{n}, \tilde{I}_{j}^{n}=\frac{1}{2}\left(I_{j}^{n}+I_{j-1}^{n}\right) & \text { for } \gamma>0, \\
\Delta I_{j}^{n}=I_{j+1}^{n}-I_{j}^{n}, \tilde{I}_{j}^{n}=\frac{1}{2}\left(I_{j}^{n}+I_{j+1}^{n}\right) & \text { for } \gamma<0 .
\end{array}
$$

When computing the phase difference $\Delta \phi_{j}^{n}$, it of crucial importance to remember that phase is given only modulo $2 \pi$. In here, we have obtained reliable and stable results by ensuring that the smallest possible difference $\Delta \phi_{j}^{n}$ modulo $2 \pi$ is applied in (28b). Using the auxiliary variable

$$
\Delta \theta_{j}^{n}= \begin{cases}\phi_{j}^{n}-\phi_{j-1}^{n}, & \text { for } \gamma>0, \\ \phi_{j+1}^{n}-\phi_{j}^{n}, & \text { for } \gamma<0\end{cases}
$$

and $\Delta \tau_{j}^{n}=\min \left\{\left|\Delta \theta_{j}^{n}\right|,\left|\Delta \theta_{j}^{n}+2 \pi\right|,\left|\Delta \theta_{j}^{n}-2 \pi\right|\right\}$ we evaluate $\Delta \phi_{j}^{n}$ as

$$
\Delta \phi_{j}^{n}= \begin{cases}\Delta \theta_{j}^{n}, & \text { if }\left|\Delta \theta_{j}^{n}\right|=\Delta \tau_{j}^{n}, \\ \Delta \theta_{j}^{n}+2 \pi, & \text { if }\left|\Delta \theta_{j}^{n}+2 \pi\right|=\Delta \tau_{j}^{n}, \\ \Delta \theta_{j}^{n}-2 \pi, & \text { if }\left|\Delta \theta_{j}^{n}-2 \pi\right|=\Delta \tau_{j}^{n} .\end{cases}
$$

The scheme (28) is of first-order accuracy and thereby entirely free of producing numerical oscillations in the approximation of (19) provided that the stability condition

$$
\max _{j \in\{-N, \ldots, N-1\}}\left\{I_{j}^{n}\right\} 3 \gamma S \frac{h}{\Delta T} \leq 1
$$

is satisfied. Our present implementation guarantees (32) under all circumstances by having the ability to adaptively take $k$ steps with step size $\Delta z$ with $h=k \Delta z$ within the central, nonlinear sub-step of the splitting method (15) when required. Note, however, that for the computations in Section IV the stability conditions (32) was always already satisfied for $k=$ 1.

To complete the algorithmic description we remark that we set $I_{j}^{0}:=\left|A_{j}^{*}\right|^{2}$ and $\phi_{j}^{0}:=\arg \left(A_{j}^{*}\right)$ after sub-step (15a) and compute $A_{j}^{* *}=\sqrt{I_{j}^{k}} e^{i \phi_{j}^{k}}$ before step (15c). Periodic boundary conditions could be implemented by one layer of halo points, cf. (11). But note that thanks to the directional dependence, inherent to (28) to (30), it suffices to update only the upstream halo point, that is the one with index $j=-N-1$ for $\gamma>0$ and the one with $j=N$ in case $\gamma<0$ before applying the upwind scheme. 


\section{High-resolution upwind scheme}

To enable overall second-order numerical accuracy of the fractional step method (15), in case the solution is smooth and differentiable, it is necessary to extend the homogeneous nonlinear update (24) to a high-resolution scheme. Highresolution upwind schemes perform an on-the-fly point-wise smoothness analysis and blend central differences with onesided difference quotients. As will be seen below, highresolution methods can vary significantly in construction; yet, they have in common that special functions, called limiters, are required for smoothness analysis. ${ }^{2}$

A high-resolution upwind method readily applicable to (24) is the Wave Propagation Method of LeVeque [17], [19] that is specially designed for quasilinear hyperbolic systems. This method reads

$$
\begin{array}{r}
\mathbf{q}_{j}^{n+1}=\mathbf{q}_{j}^{n}-\frac{h}{\Delta T}\left(\hat{\mathbf{M}}^{-} \Delta \mathbf{q}_{j+1 / 2}^{n}+\hat{\mathbf{M}}^{+} \Delta \mathbf{q}_{j-1 / 2}^{n}\right) \\
-\frac{h}{\Delta T}\left(\tilde{\mathbf{F}}_{j+\frac{1}{2}}^{n}-\tilde{\mathbf{F}}_{j-\frac{1}{2}}^{n}\right)
\end{array}
$$

with $\tilde{\mathbf{F}}$ denoting special second-order corrections terms (derived with recourse to the Lax-Wendroff scheme) given as

$$
\tilde{\mathbf{F}}_{j+\frac{1}{2}}^{n}=\frac{1}{2} \sum_{m=1}^{2}\left|\hat{\lambda}_{j+\frac{1}{2}}^{m}\right|\left(1-\frac{h}{\Delta T}\left|\hat{\lambda}_{j+\frac{1}{2}}^{m}\right|\right) \tilde{\mathcal{W}}_{j+\frac{1}{2}}^{m} .
$$

In the latter, $\tilde{\mathcal{W}}_{j+\frac{1}{2}}^{m}$ denotes a limited version of the wave $\mathcal{W}_{m}:=a_{m} \hat{\mathbf{r}}_{m}$ associated to each eigenvalue specified as

$$
\tilde{\mathcal{W}}_{j+\frac{1}{2}}^{m}=\Phi\left(\Theta_{j+\frac{1}{2}}^{m}\right) \mathcal{W}_{j+\frac{1}{2}}^{m} .
$$

The function $\Phi(\cdot)$ in (35) refers to a limiter, for instance the Minmod function $\Phi(r)=\max (0, \min (r, 1))$, and $\Theta_{j+\frac{1}{2}}^{m}$ is evaluated as the ratio of neighboring wave strengths $a_{m}$ as

$$
\Theta_{j+\frac{1}{2}}^{m}= \begin{cases}a_{j-\frac{1}{2}}^{m} / a_{j+\frac{1}{2}}^{m}, & \hat{\lambda}_{j+\frac{1}{2}}^{m} \geq 0, \\ a_{j+\frac{3}{2}}^{m} / a_{j+\frac{1}{2}}^{m}, & \hat{\lambda}_{j+\frac{1}{2}}^{m}<0 .\end{cases}
$$

Note that (34) to (36) treat each pair of eigenvalue and eigenvector separately, which is also called characteristic limiting. Characteristic limiting can produce high quality results for certain hyperbolic equations [17], [19]; however, in the case of (19) this approach tends to create non-negligible numerical oscillations when the intensity $I$ is close to zero. This issue is due to the fact that $\hat{I}$ appears in (27) in the denominator.

Instead of using characteristic limiting of the waves $\mathcal{W}_{m}$ we have developed a special MUSCL ${ }^{3}$-type slope limiting of the solution vector $\mathbf{q}$. Originally proposed by van Leer for hyperbolic equations in conservation law form [20], application

\footnotetext{
${ }^{2}$ Note that limiter functions cannot be chosen arbitrarily but need to satisfy certain mathematical requirements to ensure that the approximation from a specific high-resolution scheme is always free of numerical over- and undershots due to the blending procedure. We refer the reader to [17], [18] for proofs that all limiters used in here have the required properties and result in total variation diminishing (TVD) schemes when applied in the Wave Propagation Method or the MUSCL slope reconstruction approach.

${ }^{3}$ MUSCL stands for Monotone Upstream-centered Schemes for Conservation Laws [20].
}

to quasilinear system is not apparent. Inspired by Ketcheson \& LeVeque [21], we formulate our high-resolution method as

$$
\mathbf{q}_{j}^{n+1}=\mathbf{q}_{j}^{n}-\frac{h}{\Delta T}\left(\hat{\mathbf{M}}^{-} \Delta \mathbf{q}_{j+1 / 2}^{\star}+\hat{\mathbf{M}}^{+} \Delta \mathbf{q}_{j-1 / 2}^{\star}+\hat{\mathbf{M}} \Delta \mathbf{q}_{j}^{\star}\right)
$$

with $\Delta \mathbf{q}_{j+1 / 2}^{\star}=\mathbf{q}_{j+1}^{l}-\mathbf{q}_{j}^{r}, \Delta \mathbf{q}_{j-1 / 2}^{\star}=\mathbf{q}_{j}^{l}-\mathbf{q}_{j-1}^{r}$, and $\Delta \mathbf{q}_{j}^{\star}=\mathbf{q}_{j}^{r}-\mathbf{q}_{j}^{l}$. Here, $\mathbf{q}_{j}^{l / r}$ refers to slope-limited values constructed for each component of $\mathbf{q}$ separately as

$$
\begin{aligned}
q_{j}^{r} & =\bar{q}_{j}+\frac{1}{4} \sigma_{j}, \\
q_{j}^{l} & =\bar{q}_{j}-\frac{1}{4} \sigma_{j}
\end{aligned}
$$

with reconstructed linear local slope

$$
\sigma_{j}=\Phi\left(\frac{\Delta_{j-\frac{1}{2}}}{\Delta_{j+\frac{1}{2}}}\right) \Delta_{j+\frac{1}{2}}+\Phi\left(\frac{\Delta_{j+\frac{1}{2}}}{\Delta_{j-\frac{1}{2}}}\right) \Delta_{j-\frac{1}{2}}
$$

with $\Delta_{j-1 / 2}=\bar{q}_{j}-\bar{q}_{j-1}, \Delta_{j+1 / 2}=\bar{q}_{j+1}-\bar{q}_{j}$. In the latter, $\Phi(\cdot)$ is again a limiter function and beside the previously mentioned Minmod function, we use in here the van Albada limiter

$$
\Phi(r)=\max \left(0,\left(r^{2}+r\right) /\left(1+r^{2}\right)\right),
$$

the van Leer limiter

$$
\Phi(r)=(r+|r|) /(1+|r|),
$$

and the Superbee limiter

$$
\Phi(r)=\max (0, \min (2 r, 1), \min (r, 2)) .
$$

To permit second-order accuracy overall, we do not utilize in (38) the discrete values from the previous step $q^{n}$ but instead intermediate values $\overline{\mathbf{q}}$ computed as

$$
\overline{\mathbf{q}}_{j}=\mathbf{q}_{j}^{n}-\frac{h}{2 \Delta T}\left(\hat{\mathbf{M}}^{-} \Delta \mathbf{q}_{j+1 / 2}^{n}+\hat{\mathbf{M}}^{+} \Delta \mathbf{q}_{j-1 / 2}^{n}\right) .
$$

The consecutive application of (43) and (37) corresponds to an explicit 2-step Runge-Kutta method in the spatial update. Finally, second-order accurate symmetric operator splitting [6], [7] is employed to integrate (23) before and after the highresolution scheme. Thanks to the simplicity of $\mathbf{s}(\mathbf{q})$, using the Euler method (25) is equivalent to an explicit 2-step RungeKutta update.

Similar to Section III-C, we express the overall resulting numerical method in concise form by inserting $\hat{\mathbf{M}}^{+}=\hat{\mathbf{M}}$, $\hat{\mathbf{M}}^{-}=0$ for $\gamma>0$ into (37) and (43). By writing $q_{j}^{r}=$ $\mathcal{R}\left(\bar{q}_{j-1}, \bar{q}_{j}, \bar{q}_{j+1}\right)$ for (38a) the scheme simplifies to

$$
\begin{aligned}
\hat{\phi}_{j}^{n} & =\phi_{j}^{n}+\frac{h}{2} \gamma I_{j}^{n}, \\
\bar{I}_{j} & =I_{j}^{n}-\frac{h}{2 \Delta T}\left[3 \gamma S \tilde{I}_{j}^{n} \Delta I_{j}^{n}\right], \\
\bar{\phi}_{j} & =\hat{\phi}_{j}^{n}-\frac{h}{2 \Delta T}\left[\gamma T_{R} \Delta I_{j}^{n}+\gamma S \tilde{I}_{j}^{n} \Delta \hat{\phi}_{j}^{n}\right], \\
I_{j}^{r} & =\mathcal{R}\left(\bar{I}_{j-1}, \bar{I}_{j}, \bar{I}_{j+1}\right), \phi_{j}^{r}=\mathcal{R}\left(\bar{\phi}_{j-1}, \bar{\phi}_{j}, \bar{\phi}_{j+1}\right), \\
I_{j}^{n+1} & =I_{j}^{n}-\frac{h}{\Delta T}\left[3 \gamma S \tilde{I}_{j}^{r} \Delta I_{j}^{r}\right], \\
\tilde{\phi}_{j}^{n+1} & =\hat{\phi}_{j}^{n}-\frac{h}{\Delta T}\left[\gamma T_{R} \Delta I_{j}^{r}+\gamma S \tilde{I}_{j}^{r} \Delta \phi_{j}^{r}\right], \\
\phi_{j}^{n+1} & =\tilde{\phi}_{j}^{n+1}+\frac{h}{2} \gamma I_{j}^{n+1},
\end{aligned}
$$


where $\tilde{I}_{j}^{n}, \Delta I_{j}^{n}$ and $\tilde{I}_{j}^{r}, \Delta I_{j}^{r}$ are computed from $I_{j}^{n}$ and $I_{j}^{r}$ according to (29a); $\Delta \hat{\phi}_{j}^{n}$ and $\Delta \phi_{j}^{r}$ are obtained from (30a) and (31). Note that we also employ (31) to compute the phase differences for (39) in the reconstruction operation (44d). Like in the first-order case, the method (44) needs to satisfy the stability condition (32). The analogous method for $\gamma<0$ is derived similarly using $q_{j}^{l}=\mathcal{L}\left(\bar{q}_{j-1}, \bar{q}_{j}, \bar{q}_{j+1}\right)$ for (38b) and (29b), (30b), and (31).

We implement periodic boundary conditions with $p=2$ halo cells, cf. (11), for the discrete values $I_{j}$ and $\phi_{j}$. Only one halo element $(p=1)$ needs to be set at the upstream side of the temporal window after applying (44a); $p=2$ halo points are required after the update by $(44 b),(44 c)$ on the upstream side, and one halo element on the downstream end. Note that the reconstruction operations (44d) need to be applied also to the innermost halo point on the upstream side, e.g., to the point $j=-N-1$ for $\gamma>0$ to allow the evaluation of difference quotients of $O\left(\Delta T^{2}\right)$ in the interior point $j=-N$ in (44e) and (44f).

Finally, we want to point out that the first-order method (28) as well as the MUSCL-based second-order scheme (44) are equally applicable for $T_{R}=0$ and especially in the singular case $S=0$, which allows deactivation of Raman scattering and/or self-steepening if desired. This convenient property is due to the fact that - thanks to eigenvalues of equal sign these schemes do not require evaluation of the eigenvectors (21) or the wave strength vector (27). Note that for $S=0$ or $\max _{j}\left\{I_{j}^{n}\right\}=0$, the stability condition (32) is trivially satisfied.

\section{NumericAl RESULTS}

In order to assess the described numerical methods we simulate the propagation of a Gaussian pulse with initial shape

$$
A(0, t)=\sqrt{P_{0}} \exp \left(-\frac{1+i C}{2} \frac{T^{2}}{T_{0}^{2}}\right) .
$$

in a homogeneous fiber. The cases considered here use parameters in the range of communication lines; yet, they are designed preliminary to test the discussed numerical methods, especially the properties of the different nonlinear suboperators of Section III. Hence, we neglect linear loss and third-order dispersion and employ $\alpha=0$ and $\beta_{3}=0$ throughout. To enforce a strong influence of the nonlinearities we use $\beta_{2}=4 \times 10^{-5} \mathrm{ps}^{2} \mathrm{~km}^{-1}$ and $\gamma=1 \mathrm{~W} / \mathrm{m}$. The central wavelength is set to $\lambda_{0}=1550 \mathrm{~nm}$, from which one computes the self-steepening parameter $S=\lambda_{0} / 2 \pi c$, with $c$ denoting the speed of light in vacuum. The parameters of the initial pulse are $P_{0}=0.625 \mathrm{~mW}, T_{0}=80 \mathrm{fs}$, and $C=0$. For this configuration, the second-order dispersion length is $L_{d}=T_{0}^{2} /\left|\beta_{2}\right|=160 \mathrm{~km}$, the nonlinear length $L_{n l}=\left(\gamma P_{0}\right)^{-1}=1.6 \mathrm{~km}$ and the approximate optical shock distance reads $z_{s}=\sqrt{e} L_{n l} \omega_{0} T_{0} /(3 \sqrt{2}) \approx 60.491 \mathrm{~km}$, cf. [1].

In what follows, we denote with CSSM the split-step method (6) with central-difference operator (10); CIAP uses the same nonlinear operator but within the interaction picture method (7). FT1 and FT2 correspond to the IAP method with the Fourier-transformation-based nonlinear operators (12) and (14), respectively. The first-order accurate upwind scheme (28)

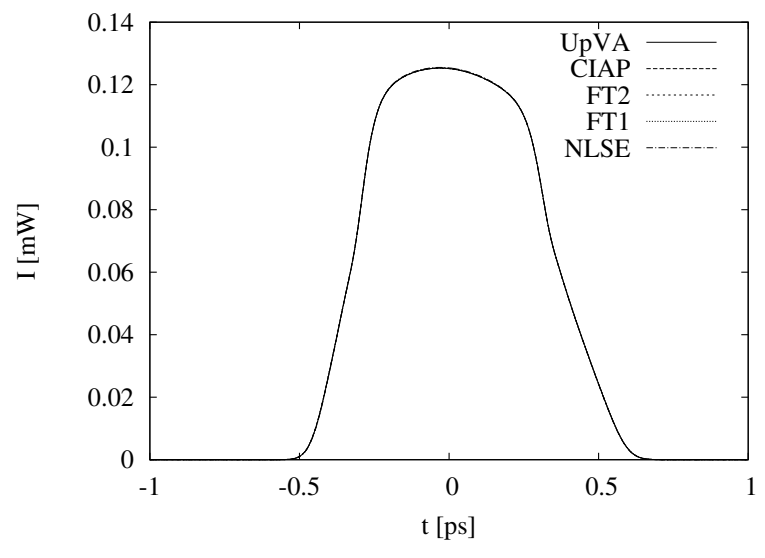

Fig. 1. Simulated signal for benchmark 1 (no Raman scattering) at $L_{\max }$ computed with different methods for $N=512$.

within the splitting method (15) is called $U p 1$; for instance the second-order accurate high-resolution operator (44) with van Albada limiter within the same method is denoted by UpVA.

\section{A. Ultra-fast dispersive pulse with self-steepening only}

In the first benchmark, the propagation distance is $L_{\max }=$ $64 \mathrm{~km}$ and the temporal window has the width $[-1 \mathrm{ps}, 1 \mathrm{ps}]$. The Raman scattering term is deactivated by setting $T_{R}=0$. Fig. 1 shows the computed solution using a temporal discretization of $2 N$ points for $N=512$ and after taking $M=800$ spatial steps of equal size of $h=80 \mathrm{~m}$. In Fig. 1 it can be seen that all schemes provide visually indistinguishable results which are also in good agreement with the solution computed with the freely available solver NLSE by Agrawal [1] at the same resolution. ${ }^{4}$ However, a detailed convergence analysis exhibits some quantitative differences. In this study, we double the temporal resolution consecutively starting from $N=128$ up to $N=8,192$ and simultaneously divide the spatial step size by a factor of 2 respectively, starting with $h=320 \mathrm{~m}$ ( $M=200$ steps). The numerical error at $L_{\max }$ is measured for the intensity of the signal in the discrete maximum norm

$$
E_{\infty}=\max _{j \in\{-N, N-1\}}\left|I_{j}-I^{\mathrm{ref}}(j \Delta T)\right|,
$$

where a highly resolved result computed with the same numerical method is used as the respective reference solution $I^{\text {ref }}$. For the schemes Up1, UpVA, CSSM, and CIAP, the respective reference result has been computed with the numerical parameters $N=16,384$ and $h=2.5 \mathrm{~m}$; the formally fourth-order accurate Fourier-based methods use a reference result with $N=4,096$ and $h=10 \mathrm{~m}$. For higher resolutions, further convergence of these methods for this benchmark appeared to be limited by round-off errors.

In Fig. 2 are shown the measured numerical errors for varying $N$; Table I provides the order of accuracy computed from two consecutive error values as

$$
\log _{2}\left(\frac{E_{\infty}(h)}{E_{\infty}\left(\frac{h}{2}\right)}\right)
$$

\footnotetext{
${ }^{4} N L S E$ is based on the second-order accurate SSFM (6) and uses an iterative procedure in approximating the nonlinear operator, cf. [1].
} 


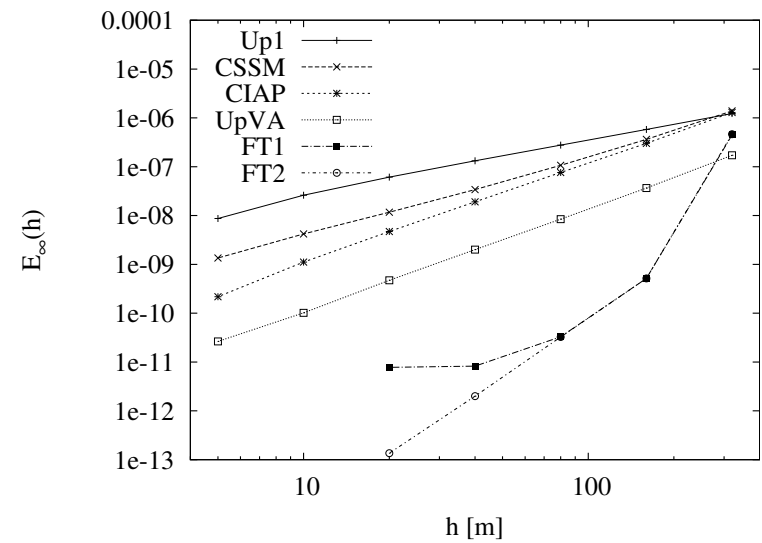

Fig. 2. Visualization of the absolute error for the investigated numerical methods for benchmark 1 .

TABLE I

ORDER OF ACCURACY COMPUTED FOR THE ERRORS SHOWN IN FIG. 2.

\begin{tabular}{|r|r||c|c|c|c|c|c|}
\hline$N$ & $h$ & Up1 & UpVA & CSSM & CIAP & FT1 & FT2 \\
\hline 256 & 160 & 1.08 & 2.23 & 1.94 & 2.11 & 9.76 & 9.81 \\
512 & 80 & 1.06 & 2.12 & 1.76 & 1.98 & 3.97 & 3.99 \\
1,024 & 40 & 1.07 & 2.07 & 1.66 & 2.00 & 2.00 & 4.02 \\
2,048 & 20 & 1.11 & 2.09 & 1.54 & 2.02 & 0.08 & 3.89 \\
4,096 & 10 & 1.23 & 2.22 & 1.48 & 2.19 & & \\
8,192 & 5 & 1.59 & 1.95 & 1.64 & 1.88 & & \\
\hline
\end{tabular}

for all six methods investigated. As it can be expected, the Up1 scheme is established as a first-order accurate discretization that shows the largest absolute errors of all methods. CSSM shows the next smaller errors, followed by CIAP. Although both methods are formally of second-order numerical accuracy, only CIAP exhibits true second-order convergence for this test case. UpVA produces absolute errors that are smaller than with any other second-order scheme and also converges reliably with second-order accuracy. Thanks to their formal fourth-order accuracy, the Fourier-based methods produce mainly even smaller absolute errors. Yet, only FT2 provides a reliable approximation that converges with fourth-order, while FTI suffers from small-scale oscillations (not specially visualized here) and consequently is lacking convergence. Given the ambiguity in scheme derivation, cf. Section III-B, this behavior gives first evidence for the lack of reliability that is characteristic for these methods.

Finally, in Table II we give the CPU times of the investigated methods for the first benchmark on a typical Linux workstation, where our implementation is in FORTRAN 90 and uses the Netlib NAPACK Fast Fourier Transformation (FFT)

TABLE II

CPU TIME IN SECONDS ON INTEL-XEON-2.80GHZ PROCESSOR FOR BENCHMARK 1 .

\begin{tabular}{|r|c|c|c||c|c|c||c|c|c|}
\hline \multicolumn{1}{|c|}{$N$} & \multicolumn{3}{|c||}{ Standard SSM } & \multicolumn{3}{c||}{ Optimized SSM } & \multicolumn{3}{|c|}{ Interaction Picture } \\
\cline { 2 - 9 } & Up1 & CSSM & UpVA & Up1 & CSSM & UpVA & CIAP & FT1 & FT2 \\
\hline 1,024 & 2.28 & 2.37 & 2.53 & 1.45 & 1.48 & 1.81 & 4.95 & 7.1 & 7.1 \\
2,048 & 8.98 & 9.34 & 10.0 & 5.62 & 5.74 & 7.02 & 19.8 & 29.3 & 28.2 \\
4,096 & 36.5 & 38.1 & 40.9 & 22.6 & 23.1 & 28.4 & 80.7 & 124 & 125 \\
8,192 & 146 & 157 & 165 & 90.0 & 92.3 & 116 & 328 & 517 & 520 \\
16,384 & 599 & 642 & 702 & 388 & 402 & 463 & 1351 & 2171 & 2160 \\
\hline
\end{tabular}

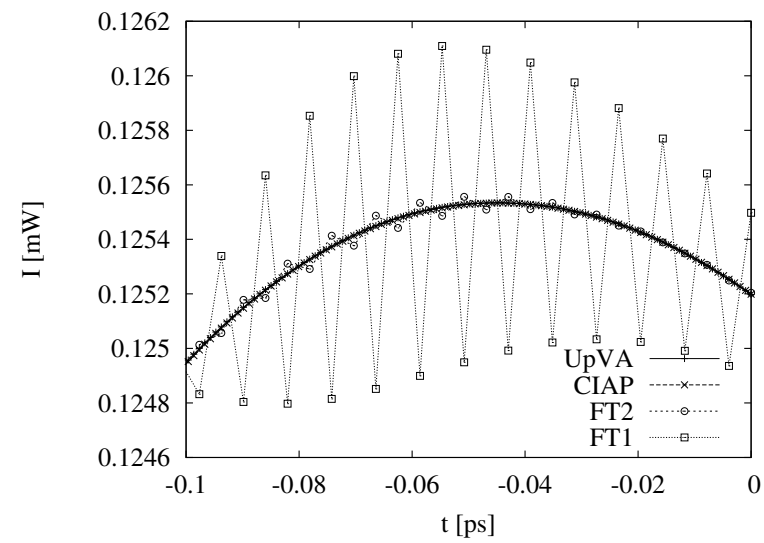

Fig. 3. Enlargement of simulated signal for benchmark 2 (Raman scattering considered). Shown resolution is $N=1,024$ for $F T 1$ and $F T 2$ and $N=$ 4, 096 for UpVA and CIAP.

routines implemented in FORTRAN 77 [22]. Here, we show the data for direct implementations of the methods (6) and (15), denoted Standard SSM (Split-Step Methods), and more compute-efficient variants (Optimized SSM), which combine the linear sub-operators from two consecutive spatial steps with step size $\frac{h}{2}$ in a single linear step with step size $h$ [1]. This approach leads to results that are identical beside roundoff errors if immediately after and before each output a linear sub-step with $\frac{h}{2}$ is taken. Table II shows that the increase in computational expense from using the high-resolution upwind method $U p V A$ is quite moderate, although the algorithm (44) involves two upwind sub-steps and additionally the slope reconstruction and limiting procedure. As it is typical for splitstep Fourier methods, the overall computational expense is primarily dominated by the number of FFT operations, the benefit from utilizing the variants denoted Optimized SSM being apparent. In general, it requires slightly less CPU time to use the optimized version of $U p V A$ at twice the resolution than using the formally fourth-order accurate IAP methods $F T 1$ or FT2.

\section{B. Ultra-fast dispersive pulse with self-steepening and Raman scattering}

The second benchmark is similar to the first but with the exception that Raman scattering is now also activated using $T_{R}=3 \mathrm{fs}$ and that we employ a temporal window of $[-4 \mathrm{ps}, 4 \mathrm{ps}]$ width. Similar to the previous case, $N$ is doubled consecutively starting from 256 up to 4,096 and $h$ is halved starting with $160 \mathrm{~m}$ down to $10 \mathrm{~m}$. While the simulated signal is qualitatively similar to Fig. 1, an enlargement of the pulse maximum in Fig. 3 shows major differences between numerical methods. While for instance UpVA and CIAP obviously approximate the same solution, both $F T 1$ and FT2 now produce unstable results and no result at all could be obtained with these schemes for the resolutions $N=2048$ and $N=4096$. Like in the previous benchmark, FT2 behaves less oscillatory than FTI. Omitted from the graphics is a comparative result from the NLSE code [1] that is also lacking robustness and reliable convergence for this test case. 


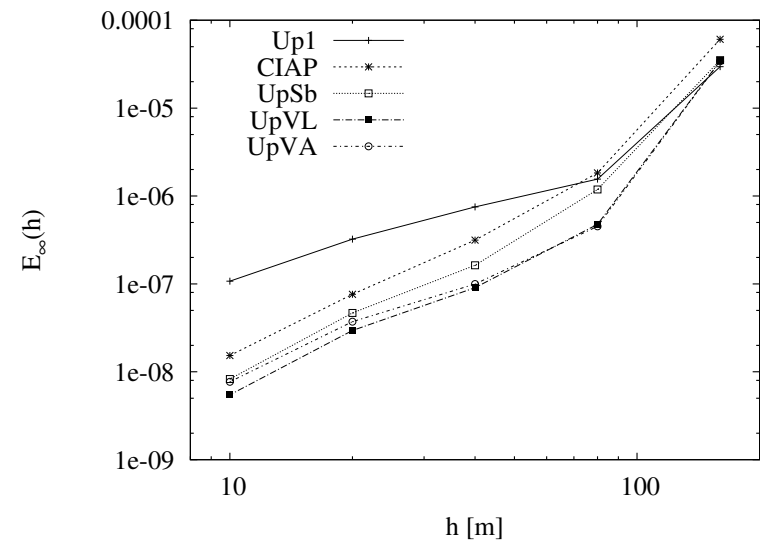

Fig. 4. Visualization of the absolute error for benchmark 2 for the converging schemes.

TABLE III

APPROXIMATION ERRORS FOR BENCHMARK 2 USING THE TWO DISCUSSED HIGH-RESOLUTION APPROACHES. ORDER OF ACCURACY $O(h)$ EVALUATED ACCORDING TO (46).

\begin{tabular}{|c|c|c|c|c|c|c|c|c|c|}
\hline \multirow{3}{*}{$N$} & \multirow{3}{*}{$\begin{array}{c}h \\
{[m]}\end{array}$} & \multicolumn{4}{|c|}{ Slope Limiting } & \multicolumn{4}{|c|}{ Wave Limiting } \\
\hline & & \multicolumn{2}{|c|}{ Superbee } & \multicolumn{2}{|c|}{ Van Leer } & \multicolumn{2}{|c|}{ Superbee } & \multicolumn{2}{|c|}{ Van Leer } \\
\hline & & $E_{\infty}$ & $O(h$ & $E_{\infty}$ & $O(h)$ & $E_{\infty}$ & $O(h)$ & $E_{\infty}$ & $O(h)$ \\
\hline 256 & 160 & $3.52 \mathrm{E}-5$ & & & & & & & \\
\hline 5 & 80 & & 4.8 & & 6.17 & 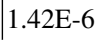 & 4.64 & & | \\
\hline 024 & 40 & $3 \mathrm{E}-8$ & 2.87 & 051 & 2.40 & 3.87E-7 & 1.87 & $3.42 \mathrm{E}-7$ & 1.20 \\
\hline 04 & 20 & $7 \mathrm{E}-8$ & 1.80 & 2.9 & 1.62 & 7.74 & 2.32 & 7.4 & 2.20 \\
\hline ,096 & 10 & $8.28 \mathrm{E}-9$ & 2.50 & $5.47 \mathrm{E}-9$ & 2.43 & $4.44 \mathrm{E}-8$ & 0.80 & $1.39 \mathrm{E}-8$ & 2.43 \\
\hline
\end{tabular}

In Fig. 4 are visualized the absolute errors of the converging numerical methods. Similar to the previous benchmark, a reference solution is used computed with the respective same numerical method but at resolution $N=8,192$ and $h=5 \mathrm{~m}$. Upl again exhibits the largest numerical errors, while the schemes with central difference nonlinear suboperator, CSSM and CIAP, now perform almost indistinguishably, with marginally better results for CIAP. Approximations with even smaller errors are produced by the methods with high-resolution operator (44), with the absolute error values dependent on the limiter function. Again, UpVA denotes this method with van Albada limiter (40), $U p V L$ refers to the same scheme but with van Leer limiter (41), and $U p S b$ uses the Superbee limiter (42). In general, $U p V L$ and $U p V A$ perform best and quite comparable; the results from $U p S b$ are slightly worse, which is generally in agreement with observations for typical hydrodynamic computations, cf. [23].

In Table III are given the absolute errors and computed approximation orders for $U p V L$ and $U p S b$. The first main column uses the proposed high-resolution approach, that is reconstruction and slope limiting by (38) to (39) within scheme (37); the second main column employs the wave propagation method (33) with wave limiting (34) to (36). The errors with the latter technique are generally substantially larger and no reliable convergence is obtained when using the Superbee limiter for this benchmark. This illustrates our previous claim that the proposed slope limiting technique is generally preferable for constructing a high-resolution sub-operator for (19).
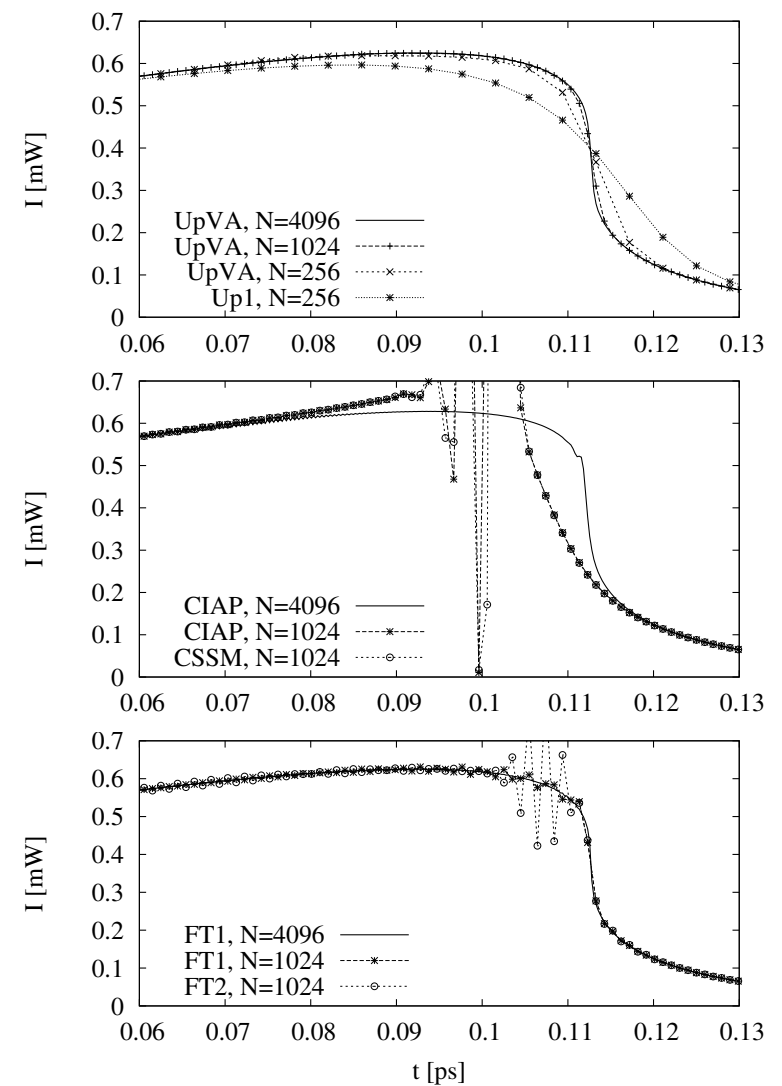

Fig. 5. Enlargement of the signal simulated with the different methods for the self-steepening benchmark 3 at different resolutions.

\section{Ultra-fast self-steepening non-dispersive pulse}

The final benchmark is intended to showcase the benefit of using shock-capturing techniques in the approximation of the nonlinearities required for ultra-fast pulse propagation, especially the self-steepening term. We employ the exact same computational parameters as in Section IV-A but deactivate dispersion and Ramann scattering by setting $\beta_{2}=0$ and $T_{R}=0$, respectively, and reduce the propagation distance to $L_{\max }=60 \mathrm{~km}$, which is slightly below the optical shock length $z_{s}$.

Fig. 5 depicts enlarged approximations of the steepening pulse front by the six methods investigated in Section IV-A under different resolutions. Since the solution is still principally smooth at $60 \mathrm{~km}$, all methods capture the correct behavior if sufficient temporal resolution is provided. For $N=4,096$ $(h=10 \mathrm{~m})$, the results from all schemes are very comparable (although CSSM and CIAP produce a marginal kink close to the front, cf. middle graphic of Fig. 5). For coarser resolutions, however, all schemes except those based on upwinding struggle with the large gradients at the steepening pulse front and produce oscillatory approximations. The methods using the central difference operator (10) now perform worst and FT2 produces larger oscillations than $F T l$. The upper graphic of Fig. 5 also visualizes the improved convergence of the highresolution method versus the first-order upwind scheme for the coarsest resolution $(N=256)$. The measured order of accuracy in the maximum norm of both upwind schemes varies 


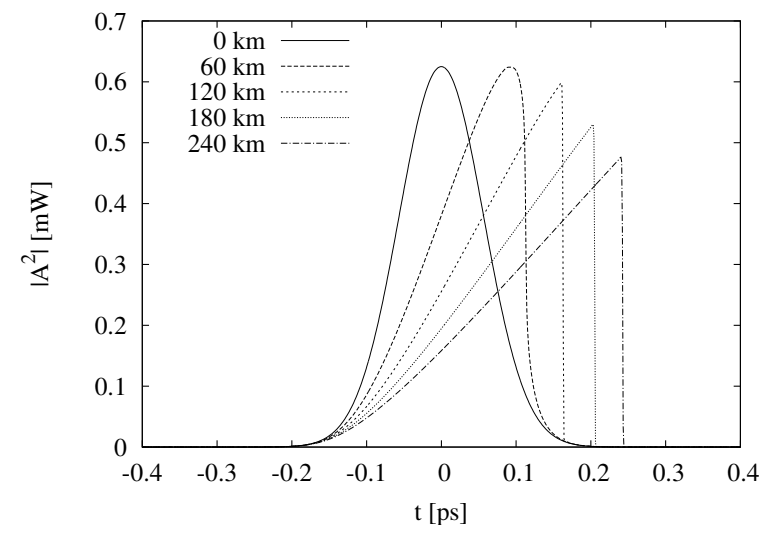

Fig. 6. Self-steepening beyond optical shock formation with $U p V A$ for $N=$ 1,024 .

around 0.5 and the absolute errors with $U p V A$ are roughly two times smaller than with $U p l$.

Numerically induced oscillations are generally increased if Ramann scattering is additionally activated. We have repeated benchmark 3 for $T_{R}=3$ fs and have obtained for the stable upwind-based schemes $U p l$ and $U p V A$ results indistinguishable to the upper graphic of Fig. 5. All other methods, however, performed significantly worse when $T_{R}=3$ fs was used (not specially visualized here). The computations broke down at all resolutions for CSSM, FT1, and FT2. Only CIAP produced a result for $N=4096$ that was still highly oscillatory. No result at all could be obtained for this benchmark with the NLSE code.

Finally, in Fig. 6 we provide snapshots from a computation with $U p V A$ that uses the same parameters as benchmark 3 but is carried on beyond the point of optical shock formation. The reliable approximation and oscillation-free handling of an even discontinuous non-dispersive signal by the proposed approach is apparent. While Fig. 6 represents an idealized scenario that is clearly outside of the physical validity of the fundamental pulse propagation equation [24], this distinction might not be so obvious for configurations characterized by very small dispersion and high nonlinearity. Especially in such situations, a computational approach for (1) that is as reliable and easy to use as the standard SSFM for pico-second pulses with $S=0$ and $T_{R}=0$, can be expected to be highly beneficial.

\section{Conclusions}

The primary difficulty in extending the split-step Fourier approach to ultra-fast pulses, as required for instance for simulating $\mathrm{Tb} / \mathrm{s}$ communication lines, lies in the development and incorporation of a nonlinear numerical sub-operator that correctly considers the mathematical nature of the nonlinear terms in the pulse propagation equation. For the special case of (1) we have shown that under the assumption of an operator splitting approach, all nonlinearities can be combined in an inhomogeneous hyperbolic system of the independent variables intensity and phase. Because of self-steepening, this hyperbolic system by itself would permit shock waves; yet, the presence of dispersion in the pulse propagation equation regularizes those. Nevertheless, solutions can encounter large gradients that present a challenge for previous split-step simulation methods, especially those based on empiric Fourier transformation of the nonlinear operator [2]. As an alternative, that is reliable even at coarse resolutions, first- and secondorder accurate shock-capturing schemes have been developed that are tailored especially to the nonlinear hyperbolic subsystem derived from (1). Integrated into a symmetric split-step Fourier method, simple propagation tests of Gaussian pulses under the influence of high nonlinearity are enough to demonstrate the robustness as well as the excellent approximation qualities of the proposed second-order accurate approach.

In our actual implementation of the described method we allow arbitrary initial signal shapes and spatially varying fiber material parameters. The code is for instance actively used to study the propagation of on-off keying modulated $\mathrm{Tb} / \mathrm{s}$ signals with super-Gaussian pulse shape in heavily dispersion-manged single-mode fibers. An extension of the approach in order to numerically solve a prototypical two-mode system for ultrafast pulses under the influence of polarization is currently under development.

Although the situation for the complex-valued pulse propagation equation (1) is significantly more complicate, we finally note that our principal idea shares resemblance with earlier work by Holden et al. [25] on numerical methods for the generalized Korteweg-de Vries equation

$$
\frac{\partial q}{\partial t}+\frac{\partial}{\partial x} f(q)+\epsilon \frac{\partial^{3}}{\partial x^{3}} q=0
$$

Holden et al. approach (47) similarly as we do for (1) and develop operator splittings that alternate between highresolution shock-capturing schemes (among others tested) for integrating the hyperbolic conservation law $\partial_{t} q+\partial_{x} f(q)=0$ and using Fourier transformation in the integration of the dispersive equation $\partial_{t} q+\epsilon \partial_{x x x} q=0$.

\section{ACKNOWLEDGMENT}

This work was supported by the Department of Defense and used resources of the Extreme Scale Systems Center at Oak Ridge National Laboratory.

\section{REFERENCES}

[1] G. P. Agrawal, Nonlinear fiber optics, 4th ed. Academic Press, 2007.

[2] V. C. Long, H. N. Viet, M. Trippenback, and K. D. Xuan, "Propagation technique for ultrashort pulses II: Numerical methods to solve the pulse propagation equation," Comp. Meth. Science Techn., vol. 14, no. 1, pp. 13-19, 2008.

[3] T. Hohage and F. Schmidt, "On the numerical solution of nonlinear Schrödinger type equations in fiber optics," Konrad-Zuse-Zentrum für Informationstechnik Berlin, Tech. Rep. ZIB-Report 02-04, Jan 2002.

[4] B. Cristiani, R. Tediosi, L. Tartara, and V. Degiorgio, "Dispersive wave generation by solitons in microstructured fibers," Optics Express, vol. 12, no. 1, pp. 124-135, 2004.

[5] A. A. Amorim, M. V. Tognetti, P. Oliveira, J. L. Silva, L. M. Bernardo, F. X. Kärtner, and H. M. Crespo, "Sub-two-cycle pulses by soliton self-compression in highly-nonlinear photonic crystal fibers," Opt. Lett., vol. 34, p. 3851, 2009.

[6] G. Strang, "On the construction and comparison of difference schemes," SIAM J. Num. Anal., vol. 5, pp. 506-517, 1968.

[7] R. Glowinski, Finite element methods for incompressible viscous flows, ser. Handbook of Numerical Analysis. Amsterdam: North-Holland, 2003, vol. IX 
[8] G. M. Muslu and H. A. Erbay, "A split-step Fourier method for the complex modified Korteweg-de Vries equation," Computers and Mathematics with Applications, vol. 45, pp. 503-514, 2003.

[9] O. V. Sinkin, R. Holzlöhner, J. Zweck, and C. R. Menyuk, "Optimization of the split-step Fourier method in modeling optical-fiber communication systems," J. LightWave Techn., vol. 21, no. 1, pp. 61-68, 2003.

[10] K. J. Blow and D. Wood, "Theoretical description of transient stimulated Raman scattering in optical fibers," IEEE J. Quantum Electronics, vol. 25, no. 12, pp. 2665-2673, 1989.

[11] J. Hult, "A fourth-order Runge-Kutta in the interaction picture method for simulating supercontinuum generation in optical fibers," J. Lightwave Techn., vol. 25, no. 12, pp. 3770-3775, 2007.

[12] X. Liu, "Adaptive higher-order split-step Fourier algorithm for simulating lightwave propagation in optical fibers," Optics Communications, vol. 282, pp. 1435-1439, 2009.

[13] P. D. Lax, "Gibbs phenomena," J. Scientific Comput., vol. 28, no. 2/3, pp. 445-449, 2006.

[14] E. Madelung, "Quantentheorie in hydrodynamischer form," Zeitschrift für Physik, vol. 40, no. 3-4, pp. 322-326, 1927.

[15] E. A. Spiegel, "Fluid dynamical form of the linear and nonlinear schrödinger equations," Physica D: Nonlinear Phenomena, vol. 1, no. 2 , pp. 236-240, 1980.

[16] J. Smoller, Shock waves and reaction-diffusion equations. New-York: Springer-Verlag, 1982.

[17] R. J. LeVeque, Finite volume methods for hyperbolic problems. Cambridge, New York: Cambridge University Press, 2002.

[18] E. F. Toro, Riemann solvers and numerical methods for fluid dynamics, 2nd ed. Berlin, Heidelberg: Springer-Verlag, 1999.

[19] R. J. LeVeque, "Wave propagation algorithms for multidimensional hyperbolic systems," J. Comput. Phys., vol. 131, no. 2, pp. 327-353, 1997.

[20] B. van Leer, "Towards the ultimate conservative difference scheme V. A second order sequel to Godunov's method," J. Comput. Phys., vol. 32, pp. 101-136, 1979

[21] D. I. Ketcheson and R. J. LeVeque, "WENOClaw: a higher order wave propagation method," in Hyperbolic problems: theory, numerics, applications. Berlin: Springer, 2008, pp. 609-616.

[22] W. Hager, Applied numerical linear algebra. Prentice-Hall, 1988.

[23] R. Deiterding, "Parallel adaptive simulation of multi-dimensional detonation structures," Ph.D. dissertation, Brandenburgische Technische Universität Cottbus, Sep 2003.

[24] F. DeMartini, C. H. Townes, T. K. Gustafson, and P. L. Kelley, "Selfsteepening of light pulses," Phys. Review, vol. 164, no. 2, pp. 312-323, 1967.

[25] H. Holden, K. H. Karlsen, and N. H. Risebro, "Operator splitting methods for generalized Korteweg-de Vries equations," J. Comput. Phys., vol. 153, pp. 203-222, 1999.

Ralf Deiterding graduated with a diploma degree in Technomathematics from the Technical University of Clausthal (Germany) in 1998 and obtained a $\mathrm{Ph} . \mathrm{D}$. in Applied Mathematics from the Brandenburg Technical University Cottbus (Germany) in 2003. Before joining Oak Ridge National Laboratory in 2006 as a staff-level mathematician, he was a Postdoctoral Scholar at the California Institute of Technology. Beside photonics simulation, he works in computational physics, computational fluid dynamics and combustion simulation.

Roland Glowinski graduated (Bsc) from Paris (France) Ecole Polytechnique in 1960, and from Paris Ecole Nationale Suprieure des Télécommunications (Ms in EE, 1963). He holds a Ph.D. in Mathematics (1970) from University P. \& M. Curie, also in Paris. He is currently Cullen Professor of Mathematics at University of Houston and an Emeritus Professor at University P. \& M. Curie, where he served as a professor before joining University of Houston in 1985. R. Glowinski is a Fellow of the American Mathematical Society (AMS), a Fellow of the Society for Industrial and Applied Mathematics (SIAM); he is also a member of the French National Academy of Sciences, of the French National Academy of Technology, and of the Academia Europaea. Glowinski works in several areas of applied and computational mathematics, mainly in computational physics and mechanics.
Hilde Oliver is a junior studying Applied Mathematics at the University of South Carolina and contributed to this work during a summer laboratory undergraduate internship at Oak Ridge National Laboratory. Beside computational physics, she is interested in mathematical biology and statistical data analysis. She graduates May 2014.

Stephen Poole Biographic information will be added before publication 